\title{
Bollwerke der Demokratie
}

VON WOLFGANG SCHROEDER

UND LUKAS HELLER

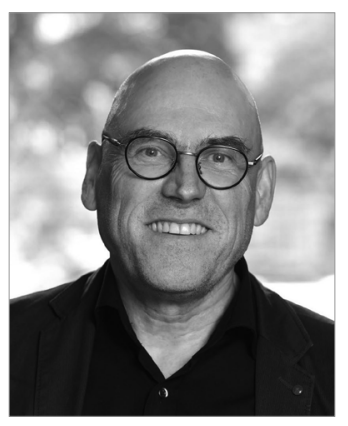

Prof. Dr. Wolfgang Schroeder ist Professor für das Politische System der Bundesrepublik an der Universität Kassel.

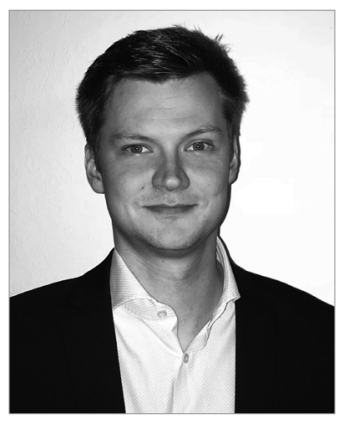

Lukas Heller ist wissenschaftlicher Mitarbeiter an der Universität Kassel. www.uni-kassel.de

Der nebenstehende Beitrag ist eine stark gekürzte Fassung des Beitrags der Autoren im Sammelband "Demokratie und Wohlfahrtspflege" (vgl. Seite 18). Dort finden sich auch Zitatbelege und Literaturhinweise.

\author{
Die Wohlfahrtsverbände repräsentieren mit ihrer \\ sozialethischen, multikulturellen und auf gleicher \\ Freiheit ausgerichteten Werteorientierung das Gegenteil \\ von dem, was rechtspopulistische Politik kennzeichnet. \\ So sind sie nicht zuletzt deshalb auch Zielscheibe \\ rechtspopulistischer Angriffe und Interventionen.
}

Die deutschen Wohlfahrtsverbände sind durch die korporatistische Tradition und Praxis des deutschen Wohlfahrtsstaates eng mit den sozialstaatlichen Aktivitäten verwoben. Dieses auf dem Subsidiaritätsprinzip aufbauende Strukturmuster schließt staatliche Direktinterventionen in den Bereich der Freien Wohlfahrtspflege aus und garantiert damit eine plurale Wohlfahrtsverbändelandschaft im Kontext eines koordinationsfähigen Gewährleistungsstaates.

Abgesichert wurde dieses Prinzip 1967 auch durch ein Urteil des Bundesverfassungsgerichts, das klarstellt, »daß der Gesetzgeber für die Verwirklichung [des Sozialstaatsprinzips nicht] nur behördliche Maßnahmen vorsehen darf; es steht ihm frei, dafür auch die Mithilfe privater Wohlfahrtsorganisationen vorzusehen«. Während damit der langjährige Versuch sozialdemokratisch regierter Städte und Länder gescheitert war, die Freie Wohlfahrtspflege zu kommunalisieren, kritisiert die »Alternative für Deutschland $(\mathrm{AfD})$ « heute die gewachsenen Beziehungen zwischen den Wohlfahrtsverbänden und dem Staat und fordert radikale Veränderungen.

Die deutschen Wohlfahrtsverbände orientieren sich an einem liberalen Gesellschaftsverständnis, das auf Vielfalt, Toleranz und Offenheit gegenüber Minderheiten ausgerichtet ist. Sie sehen ihre Aufgabe darin, "Diversität « anzuerkennen und gegen »Stereotypisierung und rassistisches Verhalten « einzutreten. Den nicht verhandelbaren Orientierungspunkt ihrer Arbeit sehen die Wohlfahrtsverbän- de in den Menschenrechten. Sie treten damit im Sinne einer »Menschenrechtsprofession « besonders für Personen ein, die von Ausgrenzung in unterschiedlichsten Formen betroffen sind. Damit engagieren sich Wohlfahrtsverbände also auch für jene, die in besonderer Weise rechtspopulistischen Aktivitäten und Anfeindungen ausgesetzt sind, wie etwa Geflüchtete.

Durch ihr vielfältiges zivilgesellschaftliches Engagement tragen Wohlfahrtsverbände zu einem demokratieorientierten Gemeinwesen bei und stärken den demokratischen Diskurs und die dort stattfindende Willensbildung. Dieses Selbstverständnis ist auch im Bewusstsein der Verbände selbst verankert. Sie suchen in diesem Sinne nach Antworten auf die Frage, wie das gesellschaftliche Zusammenleben gestaltet werden soll. Eine besondere Rolle kommt dabei den Ehrenamtlichen zu, da sie als Träger und Multiplikatoren der Positionen und der Wertebasis der jeweiligen Verbände fungieren.

\section{Falsches Image der Rechtspopulisten}

Aus der gesellschaftspolitischen Positionierung sowie der besonderen Stellung der Wohlfahrtsverbände im Wohlfahrtskorporatismus ergeben sich aus rechtspopulistischer Perspektive eine Reihe von Angriffspunkten. In gewisser Weise versuchen sich die Rechtspopulisten dabei als rechte »Watchdogs « zu inszenieren, die darauf achten, dass die Wohlfahrtsverbände mit dem ihnen anvertrauten Geld angemessen umgehen. Tatsächlich 
sind die Rechtspopulisten, inklusive der AfD, jedoch keine Watchdogs, die Verfehlungen der Wohlfahrtsverbände investigativ aufspüren und sie kontrollieren. Sie nutzen vielmehr die Erkenntnisse anderer Institutionen, wie etwa der Rechnungshöfe oder des kritischen Journalismus, um Fehlverhalten für ihre eigenen politischen Zwecke aufzugreifen. Damit bringen sie in die politisch-parlamentarische Auseinandersetzung eine Schärfe, die sich gegen die Wohlfahrtsverbände richtet, welche zuvor in der parlamentarischen Arena selten war.

Dabei greifen Rechtspopulisten die Wohlfahrtsverbände auf verschiedenen Ebenen direkt an. Gerade deshalb »springen sie nicht über jedes Stöckchen «, dass rechtspopulistische Akteure ihnen vor die Füße legen. So haben sich etwa die Spitzenverbände der Freien Wohlfahrtspflege in Thüringen darauf geeinigt, auf ein familienpolitisches Positionspapier der AfD, welches ihnen zugesandt wurde, nicht zu reagieren, um eine Rechtfertigungshaltung der Verbände zu vermeiden und der Partei keine Möglichkeit zur Inszenierung als Opfer zu bieten.

Das Ignorieren solcher Angebote, aber auch einzelner Attacken, ist fallweise möglich und sinnvoll, um die Aufmerksamkeit nicht über Gebühr zugunsten der Rechtspopulisten zu strapazieren. Dieses Verhalten ist aber nicht in allen Konstellationen möglich. Deshalb erstrecken sich die Gegenmaßnahmen von Ignorieren über Abgrenzen bis hin zum Ausgrenzen. Vermieden werden sollen öffentliche Plattformen, in denen sich Rechtspopulisten als Opfer darstellen.

\section{Rechtspopulistische Aktionen gegen Einrichtungen}

Interventionen gegenüber sozialen Diensten und Einrichtungen sind bislang auf zwei Ebenen beobachtbar: Einerseits wird versucht, ganze Einrichtungen für sich zu vereinnahmen oder bestimmte Projekte und Maßnahmen zu instrumentalisieren. Zur erfolgreichen Umsetzung dieser Fälle bedarf es einer Basis von Akteuren innerhalb dieser Einrichtungen, die diese Interventionen unterstützen und tragen.

Wo diese Anschlussfähigkeit in den Einrichtungen fehlt oder durch diese behindert wird, bleibt den Rechtspopulisten andererseits nur der Aufbau externer Gegeneinrichtungen und Gegenprojekte. So gab es verschiedene Versuche der AfD,
Leistungen in Form von Spenden medienwirksam zu vermarkten, um sie für politische Werbezwecke zu instrumentalisieren.

Die Wohlfahrtsverbände grenzen sich von diesen Angeboten deutlich ab, indem sie über die Hintergründe aufklären. Es gebe zwar lokal reale Missstände, die zu im Einzelfall auch zu Engpässen in der Versorgung führen können. Diese dürften aber nicht zur Ausgrenzung von Menschen in Notlagen und zu fremdenfeindlichen Initiativen führen.

\section{Mitarbeitende als Täter und Opfer}

Auf der Ebene der Mitarbeiterschaft hat es schon immer Einzelfälle von rassistischen oder fremdenfeindlichen Äußerungen und Verhaltensweisen gegenüber sowohl Kolleginnen und Kollegen als auch Klientinnen und Klienten gegeben.

Seitdem sich die Grenzen des Sagbaren im öffentlichen Rum verschoben haben, nehmen nach Berichten der Verbände allerdings sowohl die Intensität als auch die Häufigkeit dieser Ereignisse in den eigenen Räumen zu. Grundsätzlich gäbe es rechte Interventionen in allen Einrichtungsarten und Wohlfahrtsverbänden, bei denen in der Regel jedoch noch keine koordinierten Aktivitäten erkennbar seien. Die Bandbreite der Aktivitäten reicht von eher unterschwelligen und subtilen bis hin zur Verbreitung von offenem Hass und Hetze gegenüber Mitarbeitenden und Klientenschaft.

Die Reaktionen der Wohlfahrtsverbände bewegen sich zwischen den Dimensionen: Auseinandersetzen, Abgrenzen und Ausgrenzen. Vor allem im Falle des Bekanntwerdens von Hassposts in sozialen Medien, die mit der Wertebasis der Wohlfahrtsverbände nicht vereinbar sind oder gar rechtsextreme Hintergründe besitzen, werden Mitarbeitende auch sanktioniert, was bis zur Kündigung reichen kann. Dabei handelt es sich jedoch bislang um Einzelfälle.

Die Wohlfahrtsverbände sind zwar Tendenzbetriebe, die als solche die Möglichkeit haben, ihre Wertebindung bei den Beschäftigten einzufordern, aber per se ist eine AfD-Mitgliedschaft - solange die Partei nicht verboten ist-arbeitsrechtlich irrelevant. Zudem ist zu sehen, dass der massive Fachkräftemangel auch zu einer starken Position aufseiten der Fachkräfte führt, weil die Einrichtungen auf jede Fachkraft angewiesen sind und deshalb die
Wahrscheinlichkeit einer Kündigung bei abweichendem Verhalten eher geringer ist.

Zur Prävention suchen die Einrichtungen die eigenen Werte durch Schulungen und Handreichungen an die Mitarbeitenden herauszustellen, um deutlich zu machen, dass weder rechtspopulistische und rechtsextreme Positionen noch damit in Zusammenhang stehendes Verhalten mit einer Beschäftigung bei einem Wohlfahrtsverband vereinbar ist.

\section{Einfallstor Ehrenamtliche?}

Ein besonderes Merkmal wohlfahrtsverbandlicher Arbeit besteht darin, dass sie im Vergleich zu privaten und staatlichen Einrichtungen auf umfangreiche ehrenamtlicher Unterstützung aufbaut. Rechte Interventionen durch ehrenamtliche Unterstützer reichen von Spenden, die nur für Maßnahmen gegenüber Deutschen eingesetzt werden dürfen, Hilfsangeboten, Einflussnahmen auf inhaltliche Angebote bis hin zu Fragen, wer welche Leistungen erhalten sollte.

Zudem gibt es auch Akteure, die rechtspopulistische Ideologie bei anderen Ehrenamtlichen, den Mitarbeitenden sowie bei Klientinnen und Klienten zu verankern versuchen. In zwei Bereichen lassen sich besonders häufig solche Einflussaktivitäten beobachten: erstens in Kindertageseinrichtungen, in denen Eltern durch ihr Engagement in Elternräten und ähnlichen Gremien die Aufnahme von ausländischen Kindern zu verhindern versuchen oder beispielsweise anbieten, den Kindern "alte Hauspraktiken « zu vermitteln, wie es die Frau eines NPD-Abgeordneten in Mecklenburg-Vorpommern nannte.

Der zweite Bereich, in dem ehrenamtliches Engagement besonders augenfällig ist, ist die Obdachlosenhilfe. In diesem Feld sind vermehrt Angebote bekannt geworden, die dafür warben, sich ausschließlich um Deutsche zu kümmern.

Anders als im Bereich der hauptamtlichen Mitarbeiterschaft können Einrichtungen nicht mit arbeitsrechtlichen Maßnahmen vorgehen. In besonders gravierenden Fällen reagieren die Wohlfahrtsverbände mit Ausgrenzung, indem sie sich von ehrenamtlichen Mitgliedern und Helfenden trennen.

\section{Einfallstor Klientenschaft?}

Die Wohlfahrtsverbände und ihre Einrichtungen sind offen für alle Menschen 


\begin{tabular}{|c|c|c|c|c|}
\hline & Positionspapier/ Handreichung & Zentrale Position & $\begin{array}{l}\text { Abgren- } \\
\text { zung }\end{array}$ & $\begin{array}{l}\text { Unverein- } \\
\text { barkeit }\end{array}$ \\
\hline $\begin{array}{l}\text { Arbeiterwohlfahrt Bundes- } \\
\text { verband e.V. }\end{array}$ & $\begin{array}{l}\text { - AWO (2016a): Position des } \\
\text { Bundesverbandes der Arbei- } \\
\text { terwohlfahrt e.V. zum Um- } \\
\text { gang mit der Partei Alterna- } \\
\text { tive für Deutschland (AfD) } \\
\text { AWO (2016b): AWO gegen } \\
\text { Rassismus. Positionen und } \\
\text { Projekte }\end{array}$ & $\begin{array}{l}\text { "Sollte ein/e Mitarbeiter/in oder } \\
\text { ein Mitglied sich zu rechtsextre- } \\
\text { men, rassistischen und menschen- } \\
\text { verachtenden Äußerungen beken- } \\
\text { nen, ist eine Kündigung oder eine } \\
\text { Auflösung der Mitgliedschaft anzu- } \\
\text { streben“ (AWO 2016a). }\end{array}$ & $\checkmark$ & $\checkmark$ \\
\hline $\begin{array}{l}\text { Deutscher Caritasverband } \\
\text { e.V. }\end{array}$ & $\begin{array}{l}\text { Deutscher Caritasverband } \\
\text { (2017): Schwerpunkt: Argu- } \\
\text { mentieren gegen Rechts. In: } \\
\text { neue Caritas, 4/2017 } \\
\\
\text { Deutscher Caritasverband } \\
\text { (2016): Schwerpunkt: } \\
\text { Rechtspopulismus - Vor- } \\
\text { marsch stoppen. In: neue } \\
\text { Caritas, 20/2016. }\end{array}$ & $\begin{array}{l}\text { „Gläubige sind gefordert, sich deut- } \\
\text { lich von Rechtspopulisten abzu- } \\
\text { grenzen“ (Deutscher Caritasver- } \\
\text { band 2017) }\end{array}$ & $\checkmark$ & \\
\hline $\begin{array}{l}\text { Deutscher Paritätischer } \\
\text { Wohlfahrtsverband - Ge- } \\
\text { samtverband e.V. }\end{array}$ & $\begin{array}{l}\text { Deutscher Paritätischer } \\
\text { Wohlfahrtsverband (2019a): } \\
\text { Positionierung des Paritäti- } \\
\text { schen zu Rechtsextremismus } \\
\text { und Politik der AfD } \\
\text { Deutscher Paritätischer } \\
\text { Wohlfahrtsverband (2019b): } \\
\text { Handreichung: Umsetzung } \\
\text { des „Positionspapiers zu } \\
\text { Rechtsextremismus und Poli- } \\
\text { tik der AfD“ insbesondere in } \\
\text { Wahlkampfzeiten }\end{array}$ & $\begin{array}{l}\text { "Unser Verständnis von sozialer Ar- } \\
\text { beit ist geprägt von einer men- } \\
\text { schenrechtsorientierten Haltung, } \\
\text { die diskriminierende und men- } \\
\text { schenfeindliche Bezüge ausschließt } \\
\text { und wirksame Interventionen er- } \\
\text { möglicht" (Der Paritätische 2019a) }\end{array}$ & $\checkmark$ & \\
\hline $\begin{array}{l}\text { Diakonie Deutschland - } \\
\text { Evangelischer Bundesver- } \\
\text { band }\end{array}$ & $\begin{array}{ll} & \text { Diakonie (2018): Umgang mit } \\
\text { Rechtspopulismus. Eine } \\
\text { Handreichung für die Diako- } \\
\text { nie } \\
\text { Diakonie (2015): Demokratie } \\
\text { gewinnt! Diakonie in der Aus- } \\
\text { einandersetzung mit Diskri- } \\
\text { minierung und Rechtsextre- } \\
\text { mismus }\end{array}$ & $\begin{array}{c}\text { "Sie, [die Diakonie], wendet sich ge- } \\
\text { gen jede Form von Rassismus, Aus- } \\
\text { grenzung und gruppenbezogener } \\
\text { Menschenfeindlichkeit" (Diakonie } \\
\text { 2018) }\end{array}$ & $\checkmark$ & \\
\hline $\begin{array}{l}\text { Zentralwohlfahrtsstelle der } \\
\text { Juden in Deutschland e.V. }\end{array}$ & $\begin{array}{l}\text { ZWST et al. (2018): Keine Al- } \\
\text { ternative für Juden. Gemein- } \\
\text { same Erklärung gegen die } \\
\text { AfD. } \\
\text { - } \\
\text { nWST (2018): Antidiskrimi- } \\
\text { nierungs- und Antisemitis- } \\
\text { musprävention. In: ZWST in- } \\
\text { formiert, 4/2018. S 12-14. }\end{array}$ & $\begin{array}{l}\text { "Die AfD vertritt keinesfalls die In- } \\
\text { teressen der jüdischen Gemein- } \\
\text { schaft. Eine Partei, die außer Hass } \\
\text { und Hetze keinerlei gangbare Lö- } \\
\text { sungen für die aktuellen Herausfor- } \\
\text { derungen unserer Gesellschaft an- } \\
\text { zubieten hat, kann für niemanden } \\
\text { eine Alternative sein" (ZWST et al. } \\
\text { 2018) }\end{array}$ & $\checkmark$ & \\
\hline
\end{tabular}

Die Wohlfahrtsverbände haben in zahlreichen Stellungnahmen und Beschlüssen eindeutig Position gegen Rechtspopulismus und Rechtsextremismus bezogen.

in Not, und zwar unabhängig von ihren politischen und weltanschaulichen Präferenzen. Das schließt auch Menschen mit rechtspopulistischen Positionen ein.

Ähnlich wie im Bereich der hauptund ehrenamtlichen Mitarbeitenden gibt es seitens der Klientenschaft seit jeher, über alle Bereiche der Wohlfahrtspflege verteilt, Vorfälle, bei denen diese durch fremdenfeindliche und ähnlich abwertende, menschenfeindliche Verhaltensweisen auffallen. So sind rechte Parolen in Suppenküchen, Bewohnerinnen und Bewohner, die nicht von ausländischen Pflegekräften versorgt werden wollen und diese beleidigen oder beleidigende Ressentiments gegenüber Flüchtlingen in der Schuldnerberatung artikulieren, keine gänzlich neue Erscheinung.

Doch auch hier habe sich die Situation hinsichtlich der Zahl und der Intensität der Übergriffe in nahezu allen Einrichtungsarten verschlimmert. Besonders betroffen davon ist der Bereich der Kinder- und Jugendhilfe. Dazu gerechnet werden muss auch die Beobachtung, dass Eltern ihre Kinder motivieren, sich fremdenfeindlich und diskriminierend gegenüber anderen Kindern zu verhalten.

In der Auseinandersetzung mit abweichenden Positionen aus dem Kreis der Klientenschaft ist seitens der Einrichtungen viel Sensibilität erforderlich. In der Regel soll zunächst das Gespräch gesucht werden, um die Hintergründe des Verhaltens zu verstehen und auf dieser Basis Verhaltensänderungen bei den Klientinnen und Klienten zu ermöglichen. Hierzu sollen die entsprechenden Personen direkt eingebunden werden, um andere Erfahrungen zu machen, die sie zur Selbstreflexion und zum Lernen anregen.
Ebenfalls sei es wichtig, sich mit den Ängsten, Sorgen und dem Unmut der Klienten auseinander zu setzen. Dennoch sei auch die Abgrenzung wichtig, gemäß der mit Verweis auf die eigenen Leitbilder und Regeln die Plausibilität eines angemessenen Verhaltens eingefordert wird. Bei erfolglosen Auseinandersetzungen könne es schließlich unter bestimmten Bedingungen zu Sanktionen kommen, die zum Ausschluss der Klientinnen und Klienten führen können, indem sie aus der jeweiligen Einrichtung verwiesen werden.

\section{Fazit}

Einzelne Einrichtungen, Mitarbeitende, Ehrenamtliche und Klienten sind schon gezielt Adressaten rechtspopulistischer Unterwanderungsversuche geworden oder haben sich selbst als Träger dieser Verhaltensweisen eingebracht. Dagegen gibt es auf der verbandlichen Ebene weder inhaltliche noch personelle Berührungspunkte oder gar Schnittmengen zwischen Akteuren der Wohlfahrtsverbände und der Rechtspopulisten.

Gerade da es keine direkten Verbindungen gibt und die Wohlfahrtsverbände eine enorme Bedeutung für die Themen der Rechtspopulisten haben, sind sie immer wieder Zielpunkt außerordentlicher Kritik. So wird auch versucht, durch die medienwirksame Propagierung oder gar Gründung eigener alternativer Institutionen die etablierten WohlfahrtsverbändeEinrichtungen in ihrer wertebasierten Arbeit zu delegitimieren. Durch das Betreiben eigener sozialer Dienste - mit der Präferenz für deutsche Hilfsbedürftige - können zudem diejenigen angesprochen werden, die sich zwar für die Unterstützung sozial schwacher engagieren wollen, aber in der zunehmenden Zahl ausländischer Mitbürger oder der Flüchtlingszuwanderung eine problematische Entwicklung sehen.

Die deutschen Wohlfahrtsverbände sind ein Bollwerk gegen Rechts und deshalb ein Dorn im Auge der Rechtspopulisten. Gerade deshalb sind sie gefordert, gegenüber rechtspopulistischen Aktivitäten sensibel zu sein. 